\title{
An in vitro comparison of different adhesive strategies on the micro push-out bond strength of a glass fiber post
}

\author{
Ugur Erdemir ${ }^{1}$, Hande Sar-Sancakli ${ }^{1}$, Esra Yildiz ${ }^{1}$, Sevda Ozel ${ }^{2}$, Burak Batur ${ }^{3}$
}

\author{
${ }^{1} \mathrm{PhD}$, DDS; Department of Operative Dentistry, Faculty of Dentistry, Istanbul University (34300) Capa, Istanbul/Turkey \\ ${ }^{2} \mathrm{PhD}$; Istanbul University, Faculty of Medicine, Department of Biostatistics and Medical Informatics, (34093) Capa-Istanbul / \\ Turkey \\ ${ }^{3}$ PhD, DDS; Department of Endodontics, Faculty of Dentistry, Istanbul University (34300) Capa, Istanbul/Turkey
}

\section{Correspondence:}

Department of Operative Dentistry,

Faculty of Dentistry, Istanbul University

(34300) Capa,

Istanbul/Turkey

uerdemir@hotmail.com

Received: $18 / 06 / 2010$

Accepted: $14 / 11 / 2010$

\author{
Erdemir U, Sar-Sancakli H, Yildiz E, Ozel S, Batur B. An in vitro com- \\ parison of different adhesive strategies on the micro push-out bond \\ strength of a glass fiber post. Med Oral Patol Oral Cir Bucal. 2011 Jul \\ 1;16 (4):e626-34. \\ http://www.medicinaoral.com/medoralfree01/v16i4/medoralv16i4p626.pdf

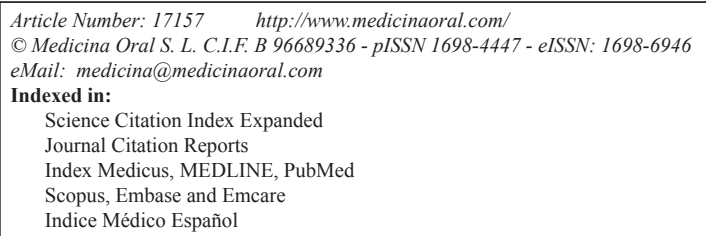

\begin{abstract}
Objectives: To evaluate in vitro the push-out bond strengths of a glass fiber post adhesively luted with self-etching resin based and self-adhesive luting cements, as well as modified application procedure of self-adhesive luting cements in combination with single step self-etch adhesives.

Materials and methods: Fifty single-rooted human maxillary central incisor teeth were endodontically treated and divided into five groups $(\mathrm{n}=10)$. Glass fiber posts (RelyX Fiber Post) were cemented with the following materials: group 1: ED Primer II/Panavia F 2.0 (PAN); group 2: RelyX Unicem (RU); group 3: Maxcem (MC); group 4: Adper Prompt L-Pop (PLP)/RelyX Unicem; group 5: Optibond all-in-one (OB)/Maxcem. Bonded specimens were cut (1-mm-thick sections) and push-out tests were performed (crosshead-speed, $0.5 \mathrm{~mm} / \mathrm{min}$ ). Data were analyzed by one-way ANOVA followed by the Tukey-HSD test for post hoc comparisons at $\alpha=0.05$.

Results: The highest bond strength was recorded for groups 4 and 2, with no statistically significant differences among them $(p>0.05)$. Group 4 showed significantly higher bond strength than group $1(p<0.001)$, group 3 $(p<0.0001)$ and group $5(p<0.0001)$. Group 1 showed significantly higher bond strength than the group $3(p<0.05)$. No statistically significant differences were observed among group 1 and group 2, group 1 and group 5, group 3 and group 5. Group 3 showed significantly lower push-out bond strength than the all other luting strategies except for the group 5.

Conclusions: The push-out bond strength values of modified application procedure of self-adhesive luting cements (RU and MC) in combination with single step self-etch dentin adhesives (PLP and OB) did not improve the pushout bond strength of fiber post when compared with those where the conventional use of self-adhesive cements.
\end{abstract}

Key words: Fiber-reinforced post, self-etch adhesives, luting cements, push-out test. 


\section{Introduction}

Posts and cores are commonly used in endodontically treated teeth suffering from excessive loss of coronal tooth structure. The selection of an appropriate restoration for endodontically treated teeth is guided by both strength and aesthetics. Available prefabricated posts were traditionally made of metal alloys, and their use were reported to have less retention, cause serious types of root fractures (1), compromise esthetic, and have the risk of corrosion or allergic reactions (2). The increasing demand for aesthetic posts and cores has led to the development of metal-free post-and-core systems, specifically usage of translucent (quartz or glass) fiber posts. The major advantages of fiber-reinforced composite (FRC) posts are that their modulus of elasticity is similar to that of dentin, and that numerous in vitro studies have shown that the posts distribute occlusal stresses more evenly in the root dentin, usually leading to fewer and more favorable root fractures, which are often repairable $(1,3)$.

Various luting agents and corresponding adhesive systems have been proposed for bonding FRC posts to root canal dentin. These materials can be divided into etch-and-rinse adhesives and self-etching systems (4). Recently, self-adhesive resin cements, which does not require any pre-treatment of dentin, was introduced.

The etch-and-rinse strategy involves the application of an acid to the dentine surface, which removes the smear layer and demineralizes the dentine to a depth of several microns (5). The acid is rinsed off using water and then a primer and an adhesive resin are applied either in separate steps or simultaneously. 'Self-etch' adhesives however, use a 'self-etching primer', a mixture of nonrinsing acidic polymerizable monomers, to simultaneously condition and prime the dentine (5). This is usually followed by the application of an adhesive resin, the so-called 'two step self-etch adhesive'. Recently, single-step self-etch adhesives have been developed for bonding composite resin to root canal dentine. Singlestep self-etch adhesives combine the primer and adhesive into one bottle enabling simultaneous demineralization and monomer penetration into the dentine (6). In an attempt to simplify procedures, a new group of resin cements have been introduced. According to their manufacturers, these products are self-adhesive, including acidic and hydrophilic monomers in their composition, which simultaneously demineralize and infiltrate enamel and dentin, resulting in strong bonding. Therefore, they require no conditioning or priming pretreatments of tooth substrate, have allowed clinicians to lute fiber posts using a simple and standardized approach that reduce technique sensitivity despite limited access to the endodontic post space $(7,8)$. Ideally, all classes of bonding agents (ie, either etch-and-rinse, or self-etch that can be in light-, self- or dual curing formulation) can be used for luting fiber posts to the root canal dentin and all different combinations of adhesive/cement systems have recently been tested (9-11).

Bond strength between post and tooth has been measured through microtensile bond strength test $(12,13)$, or pull-out (10) and push-out (4) test methods. A better estimate of the bond strength can be achieved by the push-out test compared to the conventional shear test; since the fracture occurs parallel to the dentin bonding interface in the push-out test which makes it a true shear test (13).

Thus, the aim of this study was to evaluate the push-out bond strength of a glass fiber post that was cemented with self-etching and self adhesive luting cements in three segments of teeth (the cervical, middle, and apical thirds). For self-adhesive luting cements, a modified application procedure in combination with single step self-etch adhesive systems from the same manufacturer that previously conditioned dentin were also tested. The null hypothesis tested was that the use of modified application procedures of self-adhesive luting cements would improve the push-out bond strength of fiber post rather than the other cementations strategies.

\section{Materials and Methods}

Fifty single-rooted human maxillary central incisors with fully developed apices previously extracted for periodontal reasons were selected and and stored in $0.5 \%$ chloramine- $\mathrm{T}$ at $4^{\circ} \mathrm{C}$ for a maximum of 6 months prior to use.

-Specimen preparation

The crown of each tooth was removed 2-mm coronal to the cemento-enamel junction (CEJ), using a slow-speed diamond saw (Isomet; Buehler, Lake Bluff, IL, USA) under copious water cooling to create 14-mm-long root segments. The roots were endodontically instrumented at a working length of $1 \mathrm{~mm}$ from the apex using a 35 master apical file (Maillefer, Ballaigues, Switzerland). All root canals were instrumented by the same operator. A step-back technique was used with stainless-steel K-files (Union Broach, New York, NY, USA) and Gates Glidden drills (size 2-4; Union Broach). Irrigation was performed using a $5.25 \%$ solution of $\mathrm{NaOCl}$ after each change in size of file or drill throughout the shaping process. The canals were then rinsed with distilled water, dried with paper points (Dentsply-Maillefer, Ballaigues, Switzerland), and obturated with gutta-percha cones (Dentsply-Maillefer) and sealer (AH-Plus; Dentsply DeTrey, Konstanz, Germany) using a lateral condensation technique. After the completion of endodontic treatment, cervical root canal openings were filled with a provisional restorative material (Cavit-G; 3M ESPE AG, Seefeld, Germany), and the teeth were stored in $100 \%$ humidity in black film containers for 7 days at $37^{\circ} \mathrm{C}$. 


\section{-Post luting procedures}

The gutta-percha was removed with a warm plugger (Sybron Dental Specialties, Romulus, MI, USA) leaving a minimum 4-5 mm apical seal and creating a standard post space of $9 \mathrm{~mm}$ from the coronal surface. The dowel space was drilled in each root using a calibrated drill corresponding to the conical RelyX glass Fiber Post size \#2 (3M ESPE, Seefeld, Germany). A final flushing of the canal space was accomplished using sterile water, and the canals were dried with paper points (Dentsply-Maillefer). The prepared roots were equally and randomly divided into five treatment groups $(\mathrm{n}=10)$ according to the cementation procedure performed: group 1: ED Primer II/Panavia F 2.0 [PAN] (Kuraray, Tokyo, Japan); group 2: RelyX Unicem [RU] (3M ESPE, Seefeld, Germany); group 3: Maxcem [MC] (Kerr Corporation, Orange, CA, USA); group 4: Adper Prompt L-Pop [PLP] (3M ESPE, Seefeld, Germany) was used with RU cement; group 5: Obtibond all-in-one [OB] (Kerr Corporation, Orange, CA, USA) was used with $\mathrm{MC}$ cement. Chemical compositions of all materials along with step-by-step application procedures are described in table 1 . All posts were marked at a distance of $9 \mathrm{~mm}$ from the apical end, corresponding to the length of the post space preparation and sectioned horizontally with a water-cooled diamond fissure rotary cutting instrument (Komet-Brasseler GmbH, Lemgo, Germany). After shortening, the post surfaces were cleaned with alcohol, thoroughly rinsed with distilled water, and airdried. The materials were handled in strict accordance with the manufacturer's instructions. No pretreatment was performed on the post surfaces before the cementation procedures. Following placement of the fiber post with slight pressure, in each group, excess luting cement was removed. The luting agent was light cured using a conventional quartz-tungsten-halogen light in standard mode (600 mW/cm² output; VIP; Bisco Inc. Schaumburg, IL, USA) by placing the light tip perpendicularly through the post for $40 \mathrm{~s}$. Prior to each luting procedure, the light output was measured with a light meter that was placed on the curing unit to ensure accurate light intensity.

After the cementation procedures, the coronary part of the exposed dentin was completely covered with glass ionomer cement (Fuji IX, GC Corp., Tokyo, Japan), and the teeth were stored in distilled water for 7 days at $37^{\circ} \mathrm{C}$.

\section{-Push-out bond strength tests}

Bonded specimens were sectioned horizontally with a slow-speed diamond blade (Isomet 1000, Buehler Ltd.) under water cooling to produce six 1-mm thick post/ dentin sections to yield 60 slices for each group (Fig. 1). Each specimen was marked on its coronal side with an indelible marker, and the exact length of the fiber post segments in each section was measured using a digital caliper (0.01 mm accuracy; Mitutoya, Tokyo, Japan). Each section was attached to the push-out jig with a cyanoacrylate adhesive (Zapit; Dental Ventures of America Inc, Corona, CA, USA), ensuring that the coronal surface faced the jig and that the post was centered over the hole of the jig. The post segments were loaded with a cylindrical plunger $1 \mathrm{~mm}$ in diameter centered on the post segment; contact with the surrounding dentin surface was avoided. Loads were applied in an apical-to-cervical direction with respect to individual test specimens using a universal testing machine (Shimadzu AG-1; Shimadzu Corp. Tokyo, Japan) at a crosshead speed of $0.5 \mathrm{~mm} / \mathrm{min}$ until the post was dislodged. Push-out bond strengths were calculated for each specimen by using the formula;

Debond stress $=\frac{\text { Debonding force }(N)}{A}$

where $\mathrm{A}=$ area of the post/dentin surface. Debond stress values were converted to megapascals $(\mathrm{mPa})$. The area of the post/dentin interface was determined using the formula of the surface area of a frustum (the radii on top and bottom of the post along with the height of the slice) as $A=\pi\left(r_{1}+r_{2}\right) \sqrt{ }\left(r_{1}-r_{2}\right)^{2}+h^{2}$ (Fig. 1).

-Microscopic evaluation

After testing the push-out bond strength, one specimen from cervical section of each luting strategy was prepared for scanning electron microscopy (SEM) analysis for reveal the resin tag formation. The specimens from the cervical sections of each luting system was polished 600-, 800-, 1000-, and 1200 grit silicon carbide abrasive papers (Atlas Zimpara, Istanbul, Turkey). The surface of the root slice was etched with $37 \%$ phosphoric acid (3M ESPE, St. Paul, MN, USA) for $30 \mathrm{~s}$ to remove the organic and mineral components of the dentin, rinsed with distilled water, and subsequently deproteinized by immersion in $2 \% \mathrm{NaOCl}$ solution for $120 \mathrm{~s}$. After being extensively rinsed with water and specimens were gently air dried and dehydrated with alcohol, sputter coated with gold-palladium and examined by a SEM (Jeol JSM $6360 \mathrm{LV}$ ) at a $15-\mathrm{kV}$ accelerating voltage at magnifications X1000 and photographs were taken.

-Statistical analysis

The normal distribution of the push-out bond strength data was first checked and verified using the Kolmogorov-Smirnov test. The push-out bond strength values of the 6 different post/dentin sections were pooled together for each group, and the average dentin bond strengths were calculated for the groups. Mean values were compared with one-way analysis of variance (ANOVA). As variances were homogeneous (Levene's test), one-way ANOVA was fallowed by the Tukey honestly significant difference (HSD) test for post hoc comparisons. The level of significance was set at the 0.05 probability level in all analyses, and calculations were handled using the SPSS 15.0 software (SPSS, Chicago, IL, USA). 
Table 1. Compositions and application procedures of the materials used in the present study.

\begin{tabular}{|c|c|c|c|}
\hline $\begin{array}{l}\text { Materials and } \\
\text { manufacturer }\end{array}$ & Bonding System & Composition & Application procedure \\
\hline \multicolumn{4}{|l|}{ Luting cements } \\
\hline \multirow[t]{2}{*}{$\begin{array}{l}\text { Panavia F } 2.0 \\
\text { (Kuraray Medical } \\
\text { Inc., Osaka, } \\
\text { Japan) }\end{array}$} & ED Primer II & $\begin{array}{l}\text { Base: Hydrophobic aromatic and aliphatic } \\
\text { dimethacrylate, sodium aromatic sulphinate, N,N- } \\
\text { diethanol-p-toluidine, sodium fluoride, silanized } \\
\text { barium glass sodium benzene sulphinate } \\
\text { Catalyst: MDP, hydrophobic aromatic and } \\
\text { aliphatic dimethacrylate, photoinitiator, dibenzoyl } \\
\text { peroxide, hydrophilic dimethacrylate, silanized } \\
\text { silica }\end{array}$ & $\begin{array}{l}\text { Mix equal amounts of ED primer liquids A } \\
\text { and B, apply mixture to the post space with } \\
\text { a microbrush for } 30 \mathrm{~s} \text {, gently air-dry and } \\
\text { then remove excess with paper points. Mix } \\
\text { Panavia F } 2.0 \text { paste A and B for } 20 \mathrm{~s} \text {, apply } \\
\text { the mixed paste to the post and seat it in } \\
\text { place, light cure for } 40 \mathrm{~s} \text {. }\end{array}$ \\
\hline & & $\begin{array}{l}\text { ED Primer II: HEMA, MDP, } 5-N \mathrm{MSA} \text {, } \\
\text { dimethacrylate, sodium benzene sulphinate, water, } \\
\text { accelerator }\end{array}$ & \\
\hline $\begin{array}{l}\text { Rely X Unicem } \\
\text { (3M ESPE, } \\
\text { Seefeld, Germany) }\end{array}$ & - & $\begin{array}{l}\text { Powder: Glass fillers, silica, calcium hydroxide, } \\
\text { substituted pyrimidine, peroxy compound, } \\
\text { pigments, self-cure initiators } \\
\text { Liquid: Methacrylated phosphoric esters, } \\
\text { dimethacrylates, acetate, stabilizers, self-cure } \\
\text { initiators, light-cure initiators }\end{array}$ & $\begin{array}{l}\text { Clean and dry canal with paper points and a } \\
\text { gentle stream of air, activate and mix the } \\
\text { Rely } \mathrm{X}^{\mathrm{TM}} \text { Unicem capsule for } 10-15 \mathrm{~s}, \\
\text { apply cement onto the post surface, insert } \\
\text { the post and allow the cement to cure } \\
\text { without any interference; follow by light } \\
\text { curing for } 40 \mathrm{~s} \text {. }\end{array}$ \\
\hline $\begin{array}{l}\text { Maxcem }(\text { Kerr } \\
\text { Corporation, } \\
\text { Orange, CA) }\end{array}$ & - & $\begin{array}{l}\text { Base paste: UDMA, CQ, fluoroaluminosilicate, } \\
\text { other } \\
\text { Catalyst paste: Bis-GMA, TEGDMA, GPDM, } \\
\text { barium aluminopolosilicate glass, other }\end{array}$ & $\begin{array}{l}\text { Apply the automixed paste onto the post } \\
\text { surface and seat it in place and light cure for } \\
40 \mathrm{~s} \text {. }\end{array}$ \\
\hline $\begin{array}{l}\text { Prompt L- } \\
\text { Pop/Rely X } \\
\text { Unicem (3M } \\
\text { ESPE) }\end{array}$ & $\begin{array}{l}\text { Adper Prompt } \\
\text { L-Pop }\end{array}$ & $\begin{array}{l}\text { Adper Prompt L-Pop } \\
\text { Liquid 1: Methacrylated phosphoric esters, } \\
\text { Bis-GMA, camphorquinone, stabilizers } \\
\text { Liquid 2: Water, HEMA, polyalkenoic acid } \\
\text { initiators, stabilizers }\end{array}$ & $\begin{array}{l}\text { Activate blister. Apply Prompt L-Pop to the } \\
\text { root canal, rubbing for } 15 \mathrm{~s} \text {, gently air-dry } \\
\text { and remove excess with paper points and air } \\
\text { blow to thin film, light cure for } 10 \mathrm{~s} \text {. Apply } \\
\text { Rely X Unicem cement as described above. }\end{array}$ \\
\hline $\begin{array}{l}\text { Optibond All-in- } \\
\text { one/Maxcem } \\
\text { (Kerr) }\end{array}$ & Optibond All-in-one & $\begin{array}{l}\text { Optibond All-in-one: GPDM-Self-etching } \\
\text { adhesive monomer, Comonomers including mono- } \\
\text { and di-functional methacrylate monomers, water, } \\
\text { acetone, and ethanol, CQ,nano-sized fillers, } \\
\text { including discrete nano-silica sodium } \\
\text { hexafluorosilicate }\end{array}$ & $\begin{array}{l}\text { Apply Obtibond All-in-one to the root canal } \\
\text { for } 20 \mathrm{~s} \text { with scrubbing motion, apply } \\
\text { second application for } 20 \mathrm{~s} \text { with scrubbing } \\
\text { motion, gently air dry and remove excess } \\
\text { with paper points, light cure for } 10 \mathrm{~s} \text {. } \\
\text { Apply Maxcem cement as described above. }\end{array}$ \\
\hline \multicolumn{4}{|l|}{$\begin{array}{l}\text { Fiber-reinforced } \\
\text { post }\end{array}$} \\
\hline $\begin{array}{l}\text { Rely X Fiber Post } \\
\text { (3M ESPE) }\end{array}$ & - & $\begin{array}{l}\text { Glass fibers ( } 60-70 \% \text { by weight) embedded in } \\
\text { epoxy-resin matrix containing zirconia filler }\end{array}$ & \\
\hline
\end{tabular}

MDP: 10-methacryloyloxydecyl dihydrogen phosphate; HEMA: 2-hydroxyethyl methacrylate; GPDM: glycerol phosphate dimethacrylate; UDMA: urethane dimethacrylate; TEGDMA: triethyleneglycol dimethacrylate; Bis-GMA: bisphenol A glycidyl methacrylate;

5-NMSA: N-methacryloyl 5-aminosalicylic acid; CQ: camphorquinone. 


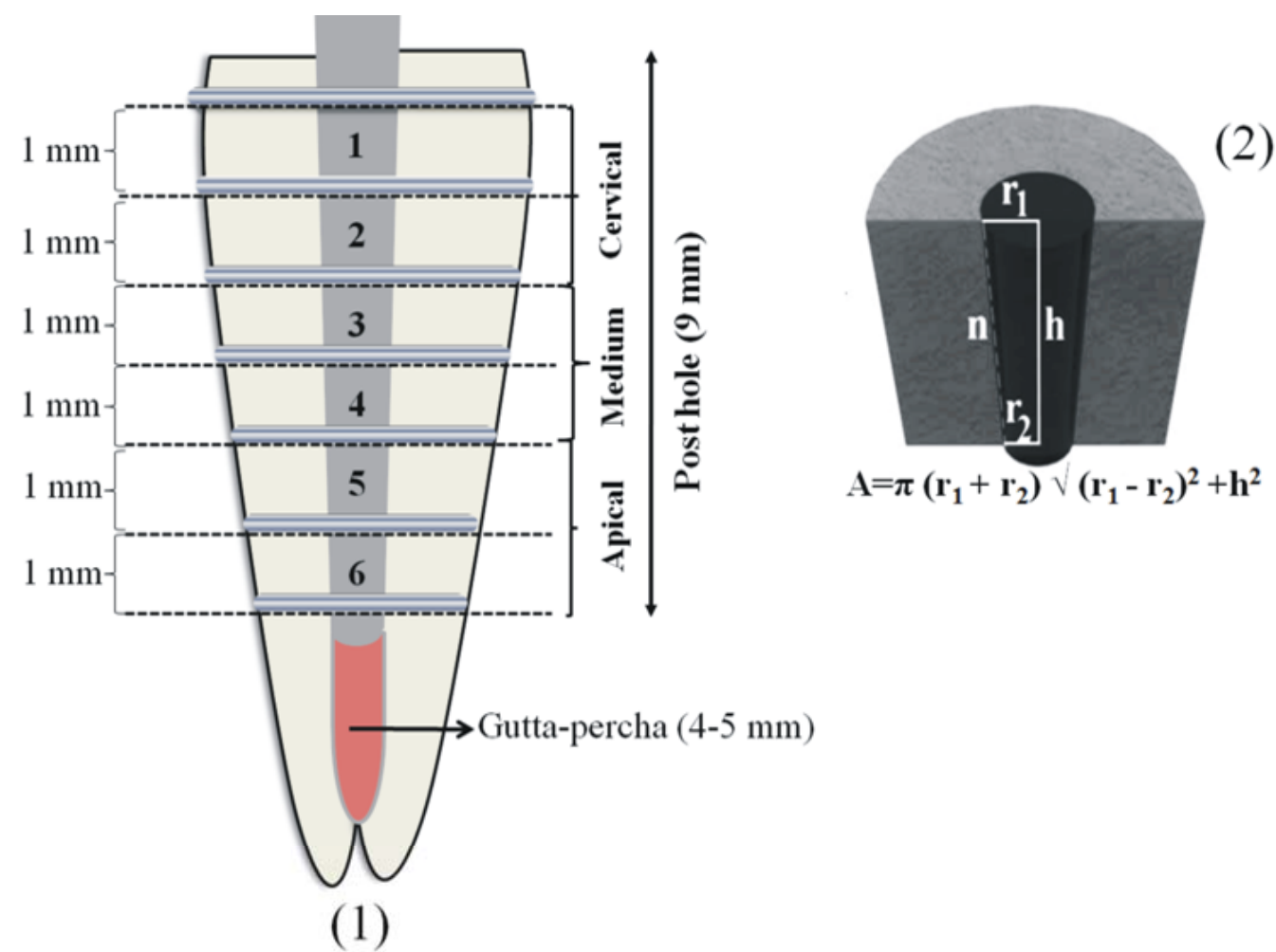

Fig. 1. Schematic view of specimen preparation for the push-out test (1) Specimen sectioning into six 1-mm thick post/dentin sections (cervical, medium, and apical); (2) The area of the post/dentin interface was determined using the formula of the surface area of a frustum: the radii of circles on top and bottom of the post along with the height of the slice $(\mathrm{h}=1 \mathrm{~mm})$.

\section{Results}

\section{-Push-out test}

The overall mean bond strength values and standard deviations for each luting strategy are presented in table 2 and figure 2. The results of the one-way ANOVA demonstrated significant differences between the luting strategies tested $(p<0.001)$. When all the regions were pooled, the PLP+RU cement $(9.91 \pm 4.82 \mathrm{MPa})$ and RU cement $(9.48 \pm 4.84 \mathrm{MPa})$ had the highest bond strengths, with no statistically significant differences among them $(\mathrm{p}=1.000)$. PLP+RU cement showed significantly higher bond strength than PAN $(8.80 \pm 4.25 \mathrm{MPa}, \mathrm{p}<0.001), \mathrm{MC}$ $(7.99 \pm 4.10 \mathrm{MPa}, \mathrm{p}<0.0001)$ and $\mathrm{OB}+\mathrm{MC}(8.18 \pm 4.07$ MPa, $\mathrm{p}<0.0001)$. PAN showed significantly higher bond strength than the $\mathrm{MC}(\mathrm{p}<0.05)$. No statistically significant differences were observed among PAN and RU, PAN and $\mathrm{OB}+\mathrm{MC}, \mathrm{MC}$ and $\mathrm{OB}+\mathrm{MC}$. MC showed significantly lower push-out bond strength than the all other luting strategies except for the $\mathrm{OB}+\mathrm{MC}$.

\section{-Microscopic observation}

Based on SEM analysis, the interfacial micromorphologies in the cervical areas of the bonded specimens are presented in figure 3. The self-etching dentin adhesive system, ED Primer II in PAN, produced numerous shorter and thinner resin tag formation extending longitudinally to the dentin wall (Fig. 3.A). However, no resin tag formations were evident in the self-adhesive luting cements RU and MC (Figs. 3.B, 3.C). For the self-adhesive luting cements, the modified application procedure in combination with single step self-etching adhesive systems (PLP, OB) produced a regular distribution with numerous and longer resin tag formation (Figs 3.D, 3.E).

\section{Discussion}

The null hypothesis of the present study was rejected because no statistically significant bond strength values were achieved with the modified application procedure of self-adhesive luting cements in combination with single step self-etch adhesives compared with those where the self-adhesive cements were used alone.

Regarding bonding effectiveness into the root canal, as determined by the push-out test, self-etching resinbased cement PAN showed lower bond strength than that of RU cement, and this results in consistent with previous reports $(4,14)$. In contrast with the present study results, Zicari et al. (15) reported higher push-out bond strength than that of RelyX Unicem (3M ESPE) ce- 


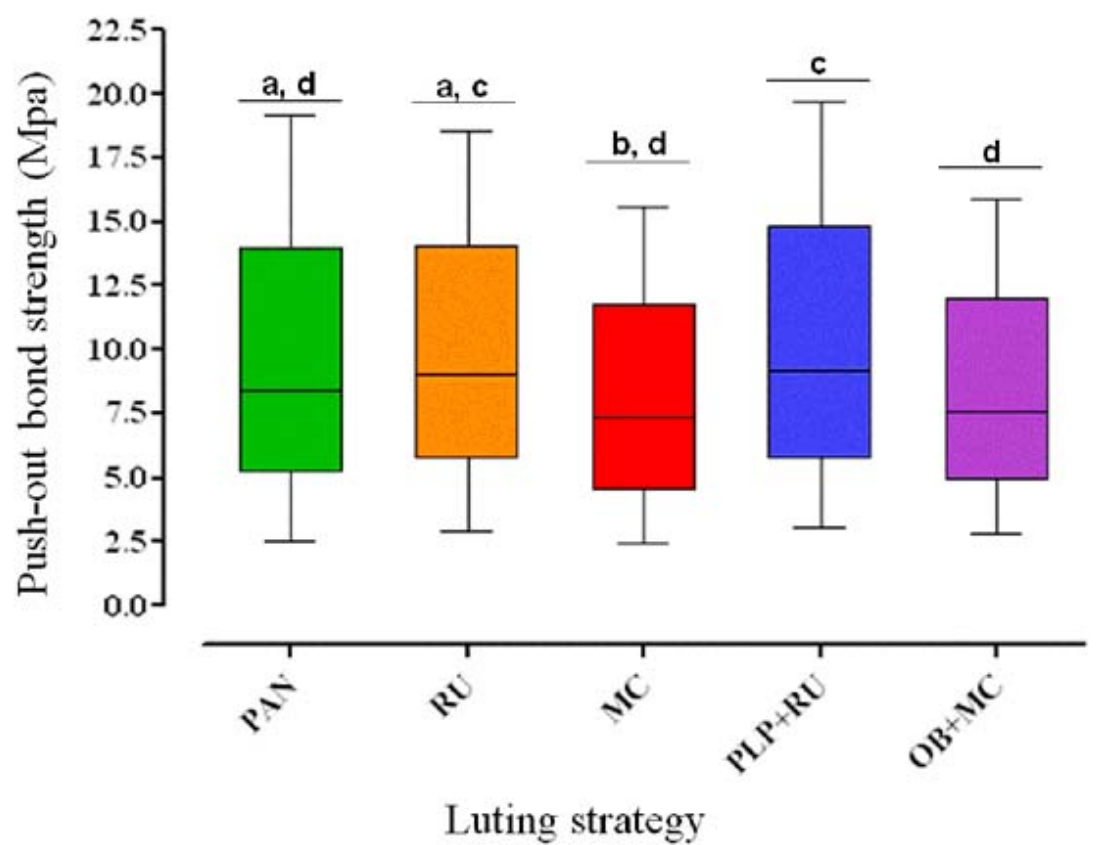

Fig. 2. Box-and-whisker plots, including medians and quartiles, indicating the push-out bond strength values of the investigated materials with respect to the when all regions were pooled. Different small letters (a, b, c, d) indicate significant differences between groups.

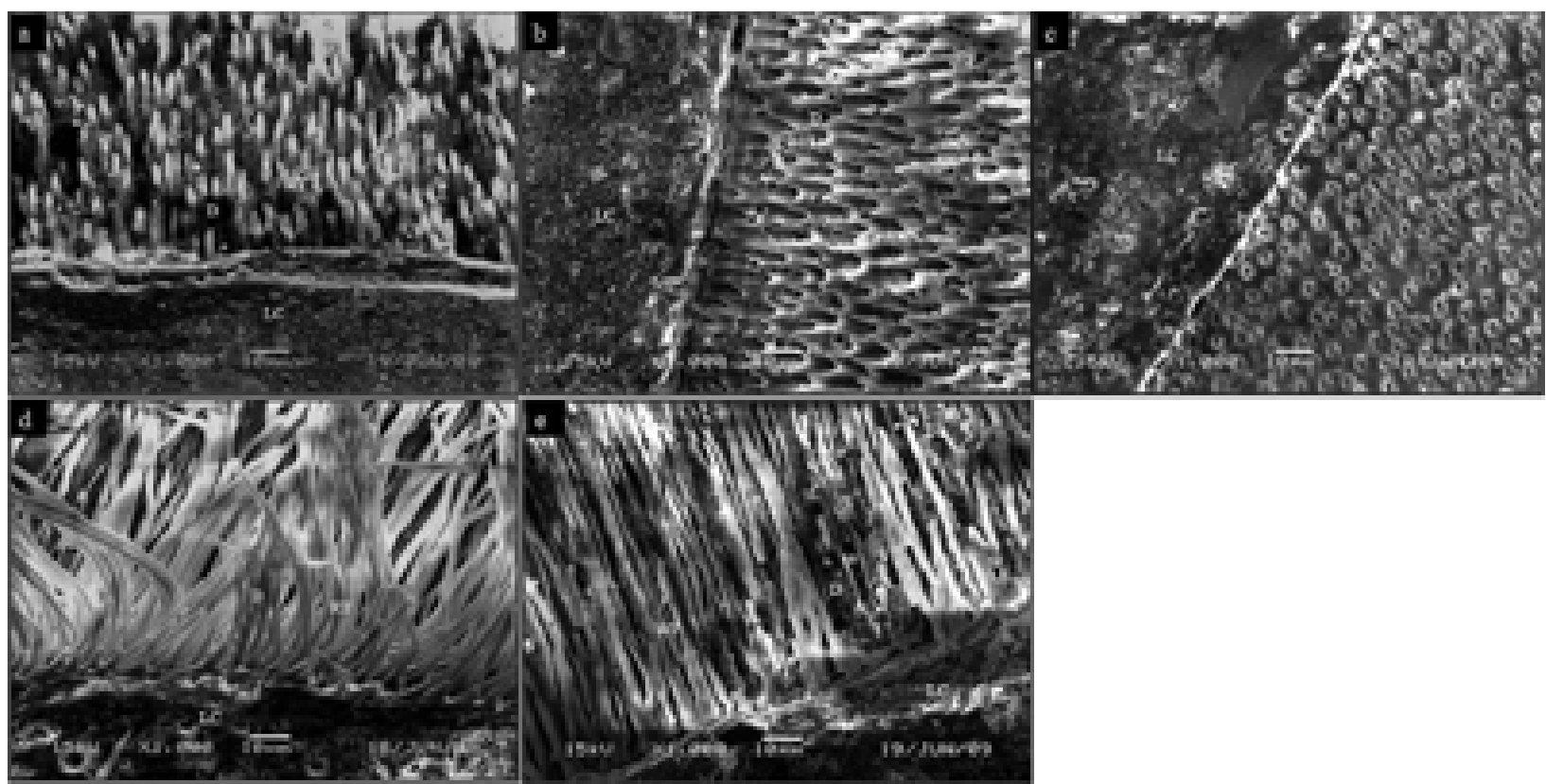

Fig. 3. Scanning electron microscopy (SEM) photographs of the interfacial micromorphologies of the bonded specimens; (a) Representative SEM photomicrograph of interface between dentin and Panavia F 2.0 luting cement in cervical root section (original magnification X1000, bar $10 \mu \mathrm{m}$ ). (LC) Luting cement, (RT) resin tag, (D) dentin, (b) Representative SEM photomicrograph of interface between dentin and RelyX Unicem luting cement in cervical root section (original magnification X1000, bar $10 \mu \mathrm{m}$ ). (LC) Luting cement, (D) dentin. (c) Representative SEM photomicrograph of interface between dentin and Maxcem luting cement in cervical root section (original magnification X1000, bar 10 $\mu \mathrm{m}$ ). (LC) Luting cement, (D) dentin. (d) Representative SEM photomicrograph of interface between dentin and modified application procedure (PLP+RU) in cervical root section (original magnification X1000, bar $10 \mu \mathrm{m}$ ). (LC) Luting cement, (RT) resin tag, (D) dentin. (e) Representative SEM photomicrograph of interface between dentin and modified application procedure $(\mathrm{OB}+\mathrm{MC})$ in cervical root section (original magnification X1000, bar $10 \mu \mathrm{m}$ ). (LC) Luting cement, (RT) resin tag, (D) dentin. 
Table 2. Overall mean push-out bond strength values (MPa) and standard deviation for each luting strategy.

\begin{tabular}{lccc}
\hline Luting strategy & No. of Specimens & Mean (MPa) & Standard Deviation \\
\hline Panavia F 2.0 & 60 & $8.80^{\text {ad }}$ & 4.46 \\
RelyX Unicem & 60 & $9.48^{\text {ac }}$ & 4.60 \\
Maxcem & 60 & $7.99^{\text {bd }}$ & 3.97 \\
PLP+RelyX Unicem & 60 & $9.91^{\mathrm{c}}$ & 5.03 \\
OB+Maxcem & 60 & $8.18^{\mathrm{d}}$ & 4.06
\end{tabular}

Different letters indicate statistically significant differences (one-way ANOVA and Tukey-HSD test, $\mathrm{p}<0.05$ )

ment. The major concern with the self-etching primers is their efficacy in infiltrating thick smear layers such as those produced during post space preparations. The etching effect of ED Primer II in PAN is related to the acidic monomer, 10-methacryloyloxydecyl dihydrogen phosphate (MDP), which does not dissolve the smear layer and results in a mild demineralization of dental tissues (16). In addition, ED Primer II consists of two Liquid bottles; Liquid A contains photoinitiators and acidic phosphate monomer (MDP), whereas Liquid B contains chemical initiators, but no MDP. When Liquid A and Liquid B were mixed, the concentrations of MDP and photoinitiators were decreased, which can result in a reduction of photopolymerization and bonding ability (5).

The bond strength obtained for RU cement was in the same range with that of PLP+RU and PAN. The bonding mechanism of this self-adhesive luting cement is claimed to be based on micromechanical retention and chemical adhesion to hydroxyapatite (7). RU exhibits a moisture tolerance due to formation of water during the neutralization reaction of phosphoric-acid methacrylate, basic fillers, and hydroxyapatite (data provided by the manufacturer). This could be an explanation for the relatively good performance of this resin cement against that of $\mathrm{MC}$ cement in the present study; since, the moisture content after rinsing the root canal is difficult to control because of the poor visibility. In agreement with the present study results, Goracci et al. (17) showed that the push-out bond strength of self-adhesive cement (RelyX Unicem; 3M ESPE) was comparable to that of self-etching resin based cement (Panavia F 2.0; Kuraray). On the other hand, this finding differed from that of a previous investigation (18), in which RelyX Unicem performed significantly worse than Panavia $\mathrm{F}$
2.0 (Kuraray), that could be explained by the omission of photoactivation of this dual-cure cement.

Pre-treatment of dentin with the single step self-etch adhesives (PLP) significantly increased the bond strength than all the other luting strategies, except for RU cement. Consistent with the present study results, in their study Hikita et al. (19) tested microtensile bond strength ( $\mu$ TBS) of PLP+RU to enamel and crown dentin, and found significantly increased bond strength to dentin. On the other hand, many laboratory $(20,21)$ and short-term clinical studies $(22,23)$ noticed an inefficient bonding effectiveness for this particular adhesive. Several explanations such as incomplete wetting and an insufficiently thick adhesive layer, phase separation between hydrophilic and hydrophobic ingredients, resultant sensitivity to hydrolysis and inhibition of polymerization of the restorative composite on top due to the high acidity of PLP at the oxygen-inhibited layer, have been advanced to explain this inconsistent bonding performance of PLP to dentin (19). The additional application of a luting composite (on top of PLP) may have served as a conventional hydrophobic resin layer removing several of the above-mentioned explanations for the relatively low bonding effectiveness of the sole use of PLP (19), thereby explaining the more favorable bonding effectiveness registered in this study.

The lowest push-out bond strength was measured for $\mathrm{MC}$ and modified application procedure of this selfadhesive cement in combination with a single step selfetch adhesive OB+MC. Similarly, Goracci et al. (24) in their investigation has been found to have a relatively poor bonding ability of this luting cement, irrespective of the tooth substrate. This results in consistent with a recently published investigation (25), in which Maxcem (Kerr Corporation) was used in combination with an 
etch-and-rinse adhesive, a two step self-etch adhesive, and a single step self-etch adhesive, and that the single step self-etch adhesive showed less favorable adhesion to root canal dentin in comparison with etch-and-rinse and the two step self-etch adhesives. However, in the present study bond-strength values increased compared with those where the MC cement was used alone. This may be explained by the same pattern has been occurred as described previously in PLP+RU cement group.

Push-out tests lead to a shear stress, which is comparable to the stress under clinical conditions (26), at the interface between dentin and luting cement, as well as between the post and luting cement (27). Considering the relative weakness of the post-root bond, Goracci et al. (28) noticed that the push-out test was a more reliable technique in the determination of bond strengths between fiber posts and post space dentin, due to the high number of premature failures occurring during specimen preparation and due to the large data distribution associated with microtensile testing. Non-uniform stress distribution is a disadvantage of the push-out test when it is performed on the thick root sections (29). To overcome this problem, original push-out test design was modified by slicing the posted root into $1-\mathrm{mm}-$ thick specimens (28). Therefore, this testing model was preferred for the present study to obtain two measurements for each third, and to simplify calculations on the bonded area.

The favorable push-out bond strengths of RU and PLP+RU cement groups may be partly explained by the fact that in the present investigation RelyX Fiber Post was used with these and all other luting strategies. According to the manufacturer's claims, this post-cement system offers both chemical compatibility and strong micromechanical post cement interlocking. Thus the adhesive/resin cement selected to lute fiber posts was chosen in accordance with the manufacturer's suggestions, except for the PAN and MC strategy: currently no specific post is recommended for these luting cements. High configuration factor ( $C$-factor) stresses may also be generated within long narrow post spaces because of rapid shrinkage with reduced flow relief during polymerization of luting cements, leading to lower bond strengths (12). The C-factor (ratio of bonded to nonbonded surfaces) associated with the posts may exceed 200 (12), and lead to numerous interfacial gaps (30). A chemically activated component of a dual-catalyst cement has been proposed to overcome the reduced strength of bonding to root canal dentin due to the shrinkage of thin layers of light cure resin composite cements during the rapid polymerization (12).

In the present study, self-etching dentin adhesive ED Primer II in PAN exhibited resin penetration into the dentin and resin tags formation in different lengths are identified. However, no evidence of resin penetration into the dentin and resin tag formation was observed in RU and MC self-adhesive luting cements. The use of single step self-etch dentin adhesives (PLP and OB) prior to application of self-adhesive luting cements exhibited long, numerous resin tags between the luting composite materials and the dentin. The resin tag observations revealed similar patterns among the single step self-etch dentin adhesives in the cervical post-dentin space regions.

Within the limitations of the present investigation, it can be concluded that the push-out bond strength values of modified application procedure of self-adhesive luting cements (RU and $\mathrm{MC}$ ) in combination with single step self-etch dentin adhesives (PLP and OB) did not improve the push-out bond strength of fiber post when compared with those where the conventional use of self-adhesive cements. In the test arrangement used, the self-adhesive approaches may be advantageous since providing satisfactory bond strength when used for luting fiber posts, which simplifies the clinical procedures. Further research may elucidate suitable combinations of adhesives/luting agents for cementing fiber posts, and clinical data are advisable for supporting the experimental data.

\section{References References with links to Crossref-DOI}

1. Torbjörner A, Karlsson S, Odman PA. Survival rate and failure characteristics for two post designs. J Prosthet Dent. 1995;73:43944.

2. Silness J, Gustavsen F, Hunsbeth J. Distribution of corrosion products in teeth restored with metal crowns retained by stainless steel posts. Acta Odontol Scand. 1979;37:317-21.

3. Ferrari M, Vichi A, Mannocci F, Mason PN. Retrospective study of the clinical performance of fiber posts. Am J Dent. 2000;13:9B$13 \mathrm{~B}$.

4. Bitter K, Meyer-Lueckel H, Priehn K, Kanjuparambil JP, Neumann $\mathrm{K}$, Kielbassa AM. Effects of luting agent and thermocycling on bond strengths to root canal dentine. Int Endod J. 2006;39:809-18.

5. Foxton RM, Nakajima M, Tagami J, Miura H. Bonding of photo and dual-cure adhesives to root canal dentin. Oper Dent. 2003;28:54351

6. Schwartz RS, Robbins JW. Post placement and restoration of endodontically treated teeth: a literature review. J Endod. 2004;30:289301.

7. Radovic I, Monticelli F, Goracci C, Vulicevic ZR, Ferrari M. Self-adhesive resin cements: a literature review. J Adhes Dent. 2008;10:251-8.

8. Monticelli F, Ferrari M, Toledano M. Cement system and surface treatment selection for fiber post luting. Med Oral Patol Oral Cir Bucal. 2008;13:E214-21.

9. Goracci C, Fabianelli A, Sadek FT, Papacchini F, Tay FR, Ferrari $\mathrm{M}$. The contribution of friction to the dislocation resistance of bonded fiber posts. J Endod. 2005;31:608-12.

10. Amaral M, Santini MF, Wandscher V, Amaral R, Valandro LF. An in vitro comparison of different cementation strategies on the pull-out strength of a glass fiber post. Oper Dent. 2009;34:443-51.

11. Ferrari M, Grandini S, Simonetti M, Monticelli F, Goracci C. Influence of a microbrush on bonding fiber post into root canals under clinical conditions. Oral Surg Oral Med Oral Pathol Oral Radiol Endod. 2002;94:627-31.

12. Bouillaguet S, Troesch S, Wataha JC, Krejci I, Meyer JM, Pashley DH. Microtensile bond strength between adhesive cements and root canal dentin. Dent Mater. 2003;19:199-205. 
13. Drummond JL, Sakaguchi RL, Racean DC, Wozny J, Steinberg AD. Testing mode and surface treatment effects on dentin bonding. J Biomed Mater Res. 1996;32:533-41.

14. Huber L, Cattani-Lorente M, Shaw L, Krejci I, Bouillaguet S. Push-out bond strengths of endodontic posts bonded with different resin-based luting cements. Am J Dent. 2007;20:167-72.

15. Zicari F, Couthino E, De Munck J, Poitevin A, Scotti R, Naert I, et al. Bonding effectiveness and sealing ability of fiber-post bonding. Dent Mater. 2008;24:967-77.

16. Molla K, Park HJ, Haller B. Bond strength of adhesive/composite combinations to dentin involving total- and self-etch adhesives. J Adhes Dent. 2002;4:171-80.

17. Goracci C, Sadek FT, Fabianelli A, Tay FR, Ferrari M. Evaluation of the adhesion of fiber posts to intraradicular dentin. Oper Dent. 2005;30:627-35.

18. De Durâo Mauricio PJ, González-López S, Aguilar-Mendoza JA, Félix S, González-Rodríguez MP. Comparison of regional bond strength in root thirds among fiber-reinforced posts luted with different cements. J Biomed Mater Res B Appl Biomater. 2007;83:36472.

19. Hikita K, Van Meerbeek B, De Munck J, Ikeda T, Van Landuyt $\mathrm{K}$, Maida T, et al. Bonding effectiveness of adhesive luting agents to enamel and dentin. Dent Mater. 2007;23:71-80.

20. Kerby RE, Knobloch LA, Clelland N, Lilley H, Seghi R. Microtensile bond strengths of one-step and self-etching adhesive systems. Oper Dent. 2005;30:195-200.

21. De Munck J, Van Meerbeek B, Satoshi I, Vargas M, Yoshida Y, Armstrong S, et al. Microtensile bond strengths of one- and twostep self-etch adhesives to bur-cut enamel and dentin. Am J Dent. 2003;16:414-20

22. Van Dijken JW. Durability of three simplified adhesive systems in Class V non-carious cervical dentin lesions. Am J Dent. 2004;17:27-32.

23. Peumans M, Kanumilli P, De Munck J, Van Landuyt K, Lambrechts P, Van Meerbeek B. Clinical effectiveness of contemporary adhesives: a systematic review of

current clinical trials. Dent Mater. 2005;21:864-81.

24. Goracci C, Cury AH, Cantoro A, Papacchini F, Tay FR, Ferrari $\mathrm{M}$. Microtensile bond strength and interfacial properties of self-etching and self-adhesive resin cements used to lute composite onlays under different seating forces. J Adhes Dent. 2006;8:327-35.

25. Topcu FT, Erdemir U, Sahinkesen G, Mumcu E, Yildiz E, Uslan I. Push-out bond strengths of two fiber post types bonded with different dentin bonding agents. J Biomed Mater Res B Appl Biomater. 2010;93:359-66.

26. Frankenberger R, Krämer N, Petschelt A. Fatigue behaviour of different dentin adhesives. Clin Oral Investig. 1999;3:11-7.

27. Van Meerbeek B, De Munck J, Yoshida Y, Inoue S, Vargas M, Vijay P, et al. Buonocore memorial lecture. Adhesion to enamel and dentin: current status and future challenges. Oper Dent. 2003;28:21535 .

28. Goracci C, Tavares AU, Fabianelli A, Monticelli F, Raffaelli O, Cardoso PC, et al. The adhesion between fiber posts and root canal walls: comparison between microtensile and push-out bond strength measurements. Eur J Oral Sci. 2004;112:353-61.

29. Asmussen E, Peutzfeldt A, Sahafi A. Finite element analysis of stresses in endodontically treated, dowel-restored teeth. J Prosthet Dent. 2005;94:321-9.

30. Boschian Pest L, Cavalli G, Bertani P, Gagliani M. Adhesive post-endodontic restorations with fiber posts: push-out tests and SEM observations. Dent Mater. 2002;18:596-602. 